\title{
An Implicit Hybrid Delay Functional Integral Equation: Existence of Integrable Solutions and Continuous Dependence
}

\author{
Ahmed M. A. El-Sayed ${ }^{1,+} \mathbb{D}$, Hind H. G. Hashem ${ }^{1,+} \mathbb{D}$ and Shorouk M. Al-Issa ${ }^{2, *, t, \ddagger}$ (D) \\ 1 Faculty of Science, Alexandria University, Alexandria 21544, Egypt; masayed@alexu.edu.eg (A.M.A.E.-S.); \\ hindhghashem@gmail.com (H.H.G.H.) \\ 2 Department of Mathematics, Faculty of Arts and Sciences, The International University of Beirut, \\ Beirut 1107, Lebanon \\ * Correspondence: shorouk.alissa@liu.edu.lb \\ + These authors contributed equally to this work. \\ $\ddagger$ Current address: Department of Mathematics, Faculty of Arts and Sciences, Lebanese International \\ University, Saida 1600, Lebanon.
}

check for

updates

Citation: El-Sayed, A.M.A.; Hashem, H.H.G.; Al-Issa, S.M. An Implicit Hybrid Delay Functional Integral Equation: Existence of Integrable Solutions and Continuous Dependence. Mathematics 2021, 9 , 3234. https://doi.org/10.3390/ math9243234

Academic Editor: Jan Awrejcewicz

Received: 1 October 2021

Accepted: 26 November 2021

Published: 14 December 2021

Publisher's Note: MDPI stays neutral with regard to jurisdictional claims in published maps and institutional affiliations.

Copyright: (C) 2021 by the authors Licensee MDPI, Basel, Switzerland. This article is an open access article distributed under the terms and conditions of the Creative Commons Attribution (CC BY) license (https:/ / creativecommons.org/licenses/by/ $4.0 /)$.

\begin{abstract}
In this work, we are discussing the solvability of an implicit hybrid delay nonlinear functional integral equation. We prove the existence of integrable solutions by using the well known technique of measure of noncompactnes. Next, we give the sufficient conditions of the uniqueness of the solution and continuous dependence of the solution on the delay function and on some functions. Finally, we present some examples to illustrate our results.
\end{abstract}

Keywords: measure of noncompactness; darbo fixed point theorem; monotonic integrable solutions; uniqueness of the solution; continuous dependence of solution

\section{Introduction}

The study of implicit differential and integral equations has received much attention over the last 30 years or so. For instance, Nieto et al. [1] have studied IFDE via the Liouville-Caputo Derivative. The integrable solutions of IFDEs has been studied in [2]. Moreover, IFDEs have recently been studied by several researchers; Dhage and Lakshmikantham [3] have proposed and studied hybrid differential equations. Zhao et al. [4] have worked at hybrid fractional differential equations and expanded Dhage's approach to fractional order. A fractional hybrid two-point boundary value problem had been studied by Sun et al. [5]. The technique of measure of noncompactness is found to be a fruitful one to obtain the existence results for a variety of differential and integral equations, for example, see [6-14].

Srivastava et al. [15] have studied the existence of monotonic integrable a.e. solution of nonlinear hybrid implicit functional differential inclusions of arbitrary fractional orders by using the measure of noncompactness technique.

Here, we investigate the existence of integrable solutions of an implicit hybrid delay functional integral equation

$$
\frac{x(t)-h(t, x(t))}{g(t, x(t))}=f_{1}\left(t, \frac{x(t)-h(t, x(t))}{g(t, x(t))}, \int_{0}^{\varphi(t)} f_{2}\left(t, s, \frac{x(s)-h(s, x(s))}{g(s, x(s))}\right) d s\right), \quad t \in[0,1] .
$$

where $\varphi:[0,1] \rightarrow[0,1], \varphi(t) \leq t$ is nondecreasing continuous function. The main tool of our study is the technique of measure noncompactness. Furthermore, we studied the continuous dependence on the delay function $\varphi$ and on the two functions $f_{1}$ and $f_{2}$.

Our article is organized as follows: In Section 2 we introduce some preliminaries. Existence results are presented in Section 3. Section 4 contains the continuous dependence of the unique solution on the delay function $\varphi$ and of the two functions $f_{1}$ and $f_{2}$. Section 5 presents two examples to verify our theorems. Lastly, conclusions are stated. 


\section{Preliminaries}

We present here some definitions and basic auxiliary results that will be needed to achieve our aim.

Let $L_{1}=L_{1}(I)$ be the class of Lebesgue integrable functions on the interval $I=[a, b]$, where $0 \leq a<b<\infty$, with the standard norm

$$
\|x\|=\int_{a}^{b}|x(t)| d t .
$$

Now, let $(E,\|\|$.$) denote an arbitrary Banach space with zero element \theta$ and $X$ a nonempty bounded subset of $E$. Moreover, denote by $B_{r}=B(\theta, r)$ the closed ball in $E$ centered at $\theta$ and with the radius $r$.

The measure of weak noncompactness defined by De Blasi [16] is given by

$$
\beta(X)=\inf \left(r>0 \text {; there exists a weakly compact subset } Y \text { of } E \text { such that } X \subset Y+B_{r}\right) \text {. }
$$

The function $\beta(X)$ possesses several useful properties that may be found in De Blasi's paper [16]. The convenient formula for the function $\beta(X)$ in $L_{1}$ was given by Appell and De Pascale [17] as follows:

$$
\beta(X)=\lim _{\epsilon \rightarrow 0}\left(\sup _{x \in X}\left(\sup \left[\int_{D}|x(t)| d t: D \subset[a, b], \text { meas } D \leq \epsilon\right]\right)\right),
$$

where the symbol meas $D$ stands for Lebesgue measure of the set $D$.

Next, we shall also use the notion of the Hausdorff measure of noncompactness $\chi$ [6] defined by

$$
\chi(X)=\inf \left(r>0 \text {; there exist a finite subset } Y \text { of } E \text { such that } X \subset Y+B_{r}\right) .
$$

In the case when the set $X$ is compact in measure, the Hausdorff and De Blasi measures of noncompactness will be identical. Namely, we have the following [16].

Theorem 1. Let $X$ be an arbitrary nonempty bounded subset of $L_{1}$. If $X$ is compact in measure, then $\beta(X)=\chi(X)$.

Now, we will recall the fixed point theorem from Banaś [18].

Theorem 2. Let $Q$ be a nonempty, bounded, closed, and convex subset of $E$, and let $T: Q \rightarrow Q$ be a continuous transformation, which is a contraction with respect to the Hausdorff measure of noncompactness $\chi$, that is, there exists a constant $\alpha \in[0,1]$ such that $\chi(T X) \leq \alpha \chi(X)$ for any nonempty subset $X$ of $Q$. Then, $T$ has at least one fixed point in the set $Q$.

We present some criterion for compactness in measure in the next section; the complete description of compactness in measure was given in Banaś [6], but the following sufficient condition will be more convenient for our purposes [6].

Theorem 3. Let $X$ be a bounded subset of $L_{1}$. Assume that there is a family of measurable subsets $\left(\Omega_{c}\right)_{0 \leq c \leq b-a}$ of the interval $(a, b)$ such that meas $\Omega_{c}=c$. If for every $c \in[0, b-a]$, and for every $x \in X$,

$$
x\left(t_{1}\right) \leq x\left(t_{2}\right), \quad\left(t_{1} \in \Omega_{c}, t_{2} \notin \Omega_{c}\right)
$$

then, the set $X$ is compact in measure.

\section{Main Results}

Now, let $I=[0,1]$ and consider the following: 
(H) (i) $f_{1}: I \times \mathbb{R} \times \mathbb{R} \rightarrow \mathbb{R}$ is a Carathéodory function which is measurable in $t \in I$, $\forall u, v \in \mathbb{R} \times \mathbb{R}$ and continuous in $u, v \in \mathbb{R} \times \mathbb{R}, \forall t \in I$.

(ii) There exists a measurable and bounded function $m_{1}: I \rightarrow I$ and nonnegative constant $b_{1}$ such that

$$
\left|f_{1}(t, u, v)\right| \leq m_{1}(t)+b_{1}(|u|+|v|), \quad t \in I .
$$

(iii) $f_{1}$ is nondecreasing on the set $I \times \mathbb{R} \times \mathbb{R}$ with respect to all the three variables, i.e., for almost all $\left(t_{1}, t_{2}\right) \in I^{2}$ such that $t_{1} \leq t_{2}$ and for all $u_{1} \leq u_{2}$ and $v_{1} \leq v_{2}$

$$
f_{1}\left(t_{1}, u_{1}, v_{1}\right) \leq f_{1}\left(t_{2}, u_{2}, v_{2}\right) .
$$

$\left(H_{2}\right) f_{2}: I \times I \times \mathbb{R} \rightarrow \mathbb{R}$ is a Carathéodory function, and a continuous function $m_{2}$ : $I \times I \rightarrow \mathbb{R}$, and nonnegative constant $b_{2}$ such that such that

$$
\left|f_{2}(t, s, x)\right| \leq m_{2}(t, s)+b_{2}|x| .
$$

Moreover, $f_{2}$ is nondecreasing on the set $I \times \mathbb{R} \times \mathbb{R}$ with respect to all the three variables.

$\left(H_{3}\right) \varphi: I \rightarrow I, \varphi(t) \leq t$ is nondecreasing. function.

$\left(H_{4}\right) g: I \times \mathbb{R} \rightarrow \mathbb{R} \backslash\{0\}$ and $h: I \times \mathbb{R} \rightarrow \mathbb{R}$ satisfy the following:

(i) They are nondecreasing on the set $I \times \mathbb{R}$ with respect to both variables, i.e., for almost all $\left(t_{1}, t_{2}\right) \in I^{2}$ such that $t_{1} \leq t_{2}$ and for all $x_{1} \leq x_{2}$

$$
h\left(t_{1}, x_{1}\right) \leq h\left(t_{2}, x_{2}\right) \text {, and } g\left(t_{1}, x_{1}\right) \leq g\left(t_{2}, x_{2}\right) ，
$$

(ii) They are measurable in $t \in I$ for every $x \in \mathbb{R}$ and continuous in $x \in \mathbb{R}$ for every $t \in I$, and there exist two integrable functions $a_{i} \in L_{1}(I)$ and two positive constants $l_{i},(i=1,2$.) such that

$$
|h(t, x)| \leq\left|a_{1}(t)\right|+l_{1}|x| \text { and }|g(t, x)| \leq\left|a_{2}(t)\right|+l_{2}|x| .
$$

Let

$$
y(t)=\frac{x(t)-h(t, x(t))}{g(t, x(t))}
$$

then the integral Equation (1) can be reduced to

$$
y(t)=f_{1}\left(t, y(t), \int_{0}^{\varphi(t)} f_{2}(t, s, y(s)) d s\right), \quad t \in I
$$

where $x$ satisfies the Equation.

$$
x(t)=h(t, x(t))+y(t) g(t, x(t)), \quad t \in I .
$$

Thus, we have proved the following result.

Theorem 4. Assume that $\left(H_{1}\right)-\left(H_{3}\right)$ be satisfied and assume that $b_{1}+b_{1} b_{2}<1$. Then the Equation (6) has at least one a.e. nondecreasing solution $y \in L_{1}$.

Proof. Define the set

$$
Q_{\rho}=\left\{y \in L_{1}:\|u\|_{L_{1}} \leq \rho\right\},
$$

Consider the integral Equation (6) and define the operator

$$
\digamma y(t)=f_{1}\left(t, y(t), \int_{0}^{\varphi(t)} f_{2}(t, s, y(s)) d s\right), t \in I .
$$


Let $y \in Q_{\rho}$, then

$$
\begin{aligned}
\|\digamma y(t)\| & =\int_{0}^{1}|\digamma y(t)| d t \\
& =\int_{0}^{1}\left|f_{1}\left(t, y(t), \int_{0}^{\varphi(t)} f_{2}(t, s, y(s)) d s\right)\right| d t \\
& \leq \int_{0}^{1}\left[m_{1}(t)+b_{1} \rho+b_{1} \int_{0}^{\varphi(t)}\left|f_{2}(t, s, y(s)) d s\right|\right] d t \\
& \leq \int_{0}^{1}\left|m_{1}(t)\right| d t+b_{1} \rho+b_{1} \int_{0}^{1} \int_{0}^{\varphi(t)}\left[m_{2}(t, s)+b_{2}|y(s)| d s\right] d t \\
& \leq \int_{0}^{1}\left|m_{1}(t)\right| d t+b_{1} \rho+b_{1} \int_{0}^{1} \int_{0}^{\varphi(t)}\left[m_{2}(t, s)+b_{2}|y(s)| d s\right] d t \\
& \leq \int_{0}^{1}\left|m_{1}(t)\right| d t+b_{1} \rho+b_{1} \int_{0}^{1} \int_{0}^{1}\left[m_{2}(t, s)+b_{2}|y(s)| d s\right] d t \\
& \leq\left\|m_{1}\right\|+b_{1}\left\|m_{2}\right\|+b_{1} \rho+b_{1} b_{2}\|y\| \\
& \leq\left\|m_{1}\right\|+b_{1}\left\|m_{2}\right\|+b_{1} \rho+b_{1} b_{2} \rho \leq \rho .
\end{aligned}
$$

Hence the operator $\digamma$ maps the ball $\mathcal{B}_{\rho}$ into itself where

$$
\rho=\left(\left\|m_{1}\right\|+b_{1}\left\|m_{2}\right\|\right)\left(1-\left[b_{1}+b_{1} b_{2}\right]\right)^{-1} .
$$

Now, $Q_{\rho}$ contains all positive and nondecreasing functions a.e. on $I$. obviously the set $Q_{\rho}$ is nonempty, bounded and convex. To prove that $Q_{\rho}$ is closed we have $\left\{x_{m}\right\} \subset Q_{\rho}$, which converges strongly to $x$. Then $\left\{x_{m}\right\}$ converges in measure to $x$ and we deduce the existence of a subsequence $\left\{x_{k}\right\}$ of $\left\{x_{m}\right\}$ which converges to $x$ a.e. on $I$ (see [19]). Therefore, $x$ is nondecreasing a.e. on $I$ which means that $x \in Q_{\rho}$. Hence the set $Q_{\rho}$ is compact in measure(see Lemma 2 in [7], p. 63).

Using $\left(H_{1}\right)-\left(H_{3}\right)$, then $\digamma$ maps $Q_{\rho}$ into itself, is continuous on $Q_{\rho}$, and transforms a nondecreasing a.e. and positive function into a function with same type (see [7]).

To show that the operator $\digamma$ is a contraction with respect to the weak noncompactness measure $\beta$. Let us start by fixing $\epsilon>0$ and $X \subset Q_{\rho}$. Furthermore, if we select a measurable subset $D \subset I$ as such meas $D \leq \epsilon$, then for any $x \in X$ using the same assumptions and argument as in [6,7], we obtain

$$
\begin{aligned}
\|\digamma y\|_{L_{1}(D)} & =\int_{D}\left|f_{1}\left(t, y(t), \int_{0}^{\varphi(t)} f_{2}(t, s, y(s)) d s\right)\right| d t \\
& \leq \int_{D}\left[m_{1}(t)+b_{1}|y(t)|+b_{1} \int_{0}^{\varphi(t)}\left|f_{2}(t, s, y(s)) d s\right|\right] d t \\
& \leq \int_{D}\left(\left|m_{1}(t)\right|+b_{1}|y(t)|\right) d t+b_{1} \int_{D} \int_{0}^{\varphi(t)}\left[m_{2}(t, s)+b_{2}|y(s)| d s\right] d t \\
& \leq \int_{D}\left(\left|m_{1}(t)\right|+b_{1}|y(t)|\right) d t+b_{1} \int_{D} \int_{0}^{\varphi(t)}\left[m_{2}(t, s)+b_{2}|y(s)| d s\right] d t \\
& \leq \int_{D}\left(\left|m_{1}(t)\right|+b_{1}|y(t)|\right) d t+b_{1} \int_{D} \int_{0}^{\varphi(t)}\left[m_{2}(t, s)+b_{2}|y(s)| d s\right] d t \\
& \leq\left\|m_{1}\right\|_{L_{1}(D)}+b_{1}\|y\|_{D}+b_{1}\left\|m_{2}\right\|_{L_{1}(D)}+b_{1} b_{2}\|y\|_{L_{1}(D)} \\
& \leq\left\|m_{1}\right\|_{L_{1}(D)}+b_{1}\left\|m_{2}\right\|_{L_{1}(D)}+\left(b_{1}+b_{1} b_{2}\right)\|y\|_{L_{1}(D)} .
\end{aligned}
$$

But

$$
\lim _{\epsilon \rightarrow 0}\left\{\sup \left\{\int_{D}\left|m_{i}(t)\right| d t: D \subset I, \text { meas } D \leq \epsilon\right\}\right\}=0, \quad i=1,2
$$


Then we find

$$
\beta(\digamma y(t)) \leq\left(b_{1}+b_{1} b_{2}\right) \beta(y(t))
$$

This implies

$$
\beta(\digamma X) \leq\left(b_{1}+b_{1} b_{2}\right) \beta(X),
$$

with $\beta$ is the De Blasi measure of weak noncompactness. The set $X$ is compact in measure, so Hausdorff and De Blasi measures of noncompactness will be identical [16], then

$$
\chi(\digamma X) \leq\left(b_{1}+b_{1} b_{2}\right) \chi(X)
$$

where $\chi$ is the Hausdorff measure of noncompactness. Since $b_{1}+b_{1} b_{2}<1$, it follows, from fixed point theorem [18], that $\digamma$ is a contraction with regard to the measure of noncompactness $\chi$ and has at least one fixed point in $Q_{\rho}$ which show that Equation (6) has at least one positive a.e. nondecreasing solution $y \in L_{1}$.

\section{Solvability of Equation (4)}

In this section, the existence of a.e. nondecreasing solutions $x \in L_{1}$ for the Equation (7) will be studied

$$
x(t)=h(t, x(t))+y(t) g(t, x(t)), \quad t \in I
$$

Theorem 5. Let the assumptions $\left(H_{2}\right),\left(H_{4}\right)$ be satisfied. Let the assumptions of Theorem 4 be satisfied. Assume that $l_{1}+M l_{2}<1$. Then there is at least one a.e. nondecreasing solution $x \in L_{1}$ to (7).

Proof. Interpret the set in the form

$$
\mathcal{B}_{r}=\left\{x \in L_{1}:\|x\| \leq r\right\}
$$

and by

$$
(\mathcal{A} x)(t)=h(t, x(t))+y(t) g(t, x(t)) .
$$

Let $x \in L_{1}$ and $M=\sup _{t \in I}|y(t)|$, then by assumptions $\left(H_{2}\right)-\left(H_{4}\right)$, we find that

$$
\begin{aligned}
|(\mathcal{A} x)(t)| & =|h(t, x(t))+y(t) g(t, x(t))| \\
& \leq|h(t, x(t))|+|y(t)||g(t, x(t))| \\
& \leq\left[\left|a_{1}(t)\right|+l_{1}|x(t)|\right]+|y(t)|\left[\left|a_{2}(t)\right|+l_{2}|x(t)|\right] .
\end{aligned}
$$

Then for $t \in I$, we have

$$
\begin{aligned}
\|\mathcal{A} x\| & =\int_{0}^{1}|(\mathcal{A} x)(t)| d t \\
& \leq \int_{0}^{1}\left[\left|a_{1}(t)\right|+l_{1}|x(t)|\right] d t+M \int_{0}^{1}\left[\left|a_{2}(t)\right|+l_{2}|x(t)|\right] d t \\
& \leq\left\|a_{1}\right\|+l_{1} \int_{0}^{1}|x(t)| d t+M\left\|a_{2}\right\|+\frac{M l_{2}}{k_{2}} \int_{0}^{1}|x(t)| d t \\
& \leq\left\|a_{1}\right\|+M\left\|a_{2}\right\|+\left(l_{1}+M l_{2}\right)\|x\| .
\end{aligned}
$$

Hence $\mathcal{A}$ maps $\mathcal{B}_{r}$ into itself where

$$
r=\left(\left\|a_{1}\right\|+M\left\|a_{2}\right\|\right)\left(1-\left[l_{1}+M l_{2}\right]\right)^{-1} .
$$

Allowing $Q_{r}$ to be a subset of $\mathcal{B}_{r}$ containing all functions that are nonnegative and a.e. nondecreasing on $I$, we may conclude that $Q_{r}$ is nonempty, closed, convex, bounded, and compact in measure ([6], p. 780). Now $Q_{r}$ is a bounded subset of $L_{1}$ that contains 
all positive and nondecreasing a.e. functions on $I$, then $Q_{r}$ is compact in measure (see Lemma 2 in [7], p. 63). As a result of assumption $\left(H_{4}\right), \mathcal{A}$ maps $Q_{r}$ into itself, is continuous on $Q_{r}$, and turns a nondecreasing a.e. and positive function into a function of the same type (see [7]).

Thus, $\mathcal{A}$ is shown to be a contraction with regard to the weak noncompactness measure $\beta$. Let us start by fixing $\epsilon>0$ and $X \subset Q_{r}$. Furthermore, if we select a measurable subset $D \subset I$ as such meas $D \leq \epsilon$, then for any $x \in X$ using the same assumptions and argument as in $[6,7]$, we obtain

$$
\begin{aligned}
\|\mathcal{A} x\|_{L_{1}(D)} & =\int_{D}|(\mathcal{A} x)(t)| d t \\
& \leq \int_{D}\left[\left|a_{1}(t)\right|+l_{1}|x(t)|\right] d t+M \int_{D}\left[\left|a_{2}(t)\right|+l_{2}|x(t)|\right] d t \\
& \leq\left\|a_{1}\right\|_{L_{1}(D)}+l_{1} \int_{D}|x(t)| d t+M\left\|a_{2}\right\|_{L_{1}(D)}+M l_{2} \int_{D}|x(t)| d t \\
& \leq\left\|a_{1}\right\|_{L_{1}(D)}+M\left\|a_{2}\right\|_{L_{1}(D)}+\left(l_{1}+M l_{2}\right)\|x\|_{L_{1}(D)} .
\end{aligned}
$$

But

$$
\lim _{\epsilon \rightarrow 0}\left\{\sup \left\{\int_{D}\left|a_{i}(t)\right| d t: D \subset I, \text { meas } D \leq \epsilon\right\}\right\}=0, \quad i=1,2 .
$$

Then we find

$$
\beta(\mathcal{A x}(t)) \leq\left(l_{1}+M l_{2}\right) \beta(x(t)) .
$$

This implies

$$
\beta(\mathcal{A X}) \leq\left(l_{1}+M l_{2}\right) \beta(X),
$$

with $\beta$ is the De Blasi measure of weak noncompactness. The set $X$ is compact in measure, so Hausdorff and De Blasi measures of noncompactness will be identical [16], then

$$
\chi(\mathcal{A X}) \leq\left(l_{1}+M l_{2}\right) \chi(X)
$$

where $\chi$ is the Hausdorff measure of noncompactness. Since $l_{1}+M l_{2}<1$, it follows, from fixed point theorem [18], that $\mathcal{A}$ is a contraction with regard to the measure of noncompactness $\chi$ and has at least one fixed point in $Q_{r}$ which show that Equation (7) has at least one positive nondecreasing a.e. solution $x \in L_{1}$.

Now, we are in position to state an existence result for the hybrid implicit functional Equation (1).

Theorem 6. Let the assumptions of Theorems 4 and 5 be satisfied. Then the implicit hybrid delay functional integral Equation (1) has at least one nondecreasing a.e. solution $x \in L_{1}$ which satisfies (7) where $y \in L_{1}$ is the nondecreasing a.e. solution of (6).

\section{Continuous Dependence}

Here, we investigate the continuous dependence of the unique solution $x \in L_{1}$ on the delay function $\varphi$ and on the two functions $f_{1}$ and $f_{2}$.

\subsection{Uniqueness of the Solution}

Consider the assumptions:

$\left(H_{1}^{*}\right)$ (i) $f_{1}: I \times \mathbb{R} \times \mathbb{R} \rightarrow \mathbb{R}$ is measurable in $t \in I, \forall u, v \in \mathbb{R} \times \mathbb{R}$ and satisfies the Lipschitz condition

$$
\left|f_{1}\left(t, u_{1}, v_{1}\right)-f_{1}\left(t, u_{2}, v_{2}\right)\right| \leq b_{1}\left(\left|u_{1}-u_{2}\right|+\left|v_{1}-v_{2}\right|\right), \quad b_{1}>0 .
$$

(ii) From assumption (i) we can deduce

$$
\left|f_{1}(t, u, v)\right| \leq m_{1}(t)+b_{1}(|u|+|v|), \quad t \in I, \quad m_{1}(t)=\sup _{t \in I} f_{1}(t, 0.0) .
$$


(iii) $f_{1}$ is nondecreasing on the set $I \times \mathbb{R} \times \mathbb{R}$ with respect to all the three variables, i.e., for almost all $\left(t_{1}, t_{2}\right) \in I^{2}$ such that $t_{1} \leq t_{2}$ and for all $u_{1} \leq u_{2}$ and $v_{1} \leq v_{2}$

$$
f_{1}\left(t_{1}, u_{1}, v_{1}\right) \leq f_{1}\left(t_{2}, u_{2}, v_{2}\right) .
$$

$\left(H_{2}^{*}\right) f_{2}: I \times I \times \mathbb{R} \rightarrow \mathbb{R}$ is Lipschitz function with a Lipschitz constant $b_{2}>0$, as a result

$$
\left|f_{2}(t, s, u)-f_{2}(t, s, v)\right| \leq b_{2}|u-v| \text {. }
$$

Hence

$$
\left|f_{2}(t, s, u)\right| \leq m_{2}+b_{2}|u|, \text { where } m_{2}=\sup _{t \in I}\left|f_{2}(t, s, 0)\right| .
$$

Moreover, $f_{2}$ is nondecreasing on the set $I \times \mathbb{R} \times \mathbb{R}$ with respect to all the three variables.

$\left(H_{3}^{*}\right) g: I \times \mathbb{R} \rightarrow \mathbb{R} \backslash\{0\}$, and $h: I \times \mathbb{R} \rightarrow \mathbb{R}$, are measurable in $t \in I$ for every $x, y \in R$ as well as meet the Lipschitz condition

$$
\begin{aligned}
|h(t, u)-h(t, v)| & \leq l_{1}|u-v|, \\
|g(t, u)-g(t, v)| & \leq l_{2}|u-v|,
\end{aligned}
$$

for all $t \in I$ and $u, v \in \mathbb{R}$. Moreover $h, g$ are nondecreasing a.e. in the two arguments. Note

$$
|h(t, u)| \leq l_{1}|u(t)|+H, \quad|g(t, u)| \leq l_{2}|u(t)|+G,
$$

where $H=\sup _{t \in I}|h(t, 0)|$ and $G=\sup _{t \in I}|g(t, 0)|$.

Theorem 7. Let the assumptions of Theorem 4 be satisfied with replace conditions $\left(H_{1}\right),\left(H_{2}\right)$ by $\left(H_{1}^{*}\right),\left(H_{2}^{*}\right)$. If $b_{1}+b_{1} b_{2}<1$, Then the functional integral Equation (6) has a unique solution.

Proof. Let $y_{1}, y_{2}$ be solutions of Equation (6), then

$$
\begin{aligned}
\left|y_{1}(t)-y_{2}(t)\right| & =\left|f_{1}\left(t, y_{1}(t), \int_{0}^{\varphi(t)} f_{2}\left(t, s, y_{1}(s)\right) d s\right)-f_{1}\left(t, y_{2}(t), \int_{0}^{\varphi(t)} f_{2}\left(t, s, y_{2}(s)\right) d s\right)\right| \\
& \leq b_{1}\left|y_{1}(t)-y_{2}(t)\right|+b_{1} \int_{0}^{\varphi(t)}\left|f_{2}\left(t, s, y_{1}(s)\right)-f_{2}\left(t, s, y_{2}(s)\right)\right| d s \\
& \leq b_{1}\left|y_{1}(t)-y_{2}(t)\right|+b_{1} b_{2} \int_{0}^{\varphi(t)}\left|y_{1}(s)-y_{2}(s)\right| d s .
\end{aligned}
$$

Taking supermum for $t \in I$, we have

$$
\begin{aligned}
\left\|y_{1}-y_{2}\right\| & \leq b_{1}|| y_{1}-y_{2} \|+b_{1} b_{2} \int_{0}^{1} \int_{0}^{\varphi(t)}\left|y_{1}(s)-y_{2}(s)\right| d s d t \\
& \leq\left(b_{1}+b_{1} b_{2}\right)\left\|y_{1}-y_{2}\right\| .
\end{aligned}
$$

Therefore

$$
\left(1-\left(b_{1}+b_{1} b_{2}\right)\right)\left\|y_{1}-y_{2}\right\| \leq 0,
$$

which implies $y_{1}=y_{2}$. Hence the solution of the problem (6) is unique.

Next, we prove the following result.

Theorem 8. Let the assumptions of Theorems 5 and 7 be satisfied, with replace condition $\left(H_{3}\right)$ by $\left(H_{3}^{*}\right)$ equipped with $\left(l_{1}+M l_{2}\right)<1$. Then the solution $x \in L_{1}$ of the functional Equation (7) is unique. 
Proof. Firstly, Theorem 5 proved that the functional Equation (7) has at least one solution. Now let $x_{1}, x_{2} \in L_{1}(I)$ be two solutions of (7). Then for $t \in I$, we have

$$
\begin{aligned}
\left|x_{1}(t)-x_{2}(t)\right| & =\left|h\left(t, x_{1}(t)\right)+y(t) g\left(t, x_{1}(t)\right)-h\left(t, x_{2}(t)\right)-y(t) g\left(t, x_{2}(t)\right)\right| \\
& \leq\left|h\left(t, x_{1}(t)\right)-h\left(t, x_{2}(t)\right)\right|+|y(t)|\left|g\left(t, x_{1}(t)\right)-g\left(t, x_{2}(t)\right)\right| \\
& \leq\left|l_{1}(t)\right|\left|x_{1}(t)-x_{2}(t)\right|+|y(t)| l_{2}(t)\left|x_{1}(t)-x_{2}(t)\right| .
\end{aligned}
$$

Then for $t \in I$, and $|y(t)|<M$, we obtain

$$
\begin{aligned}
\left\|x_{1}-x_{2}\right\| & \leq l_{1} \int_{0}^{1}\left|x_{1}(t)-x_{2}(t)\right| d t+M l_{2} \int_{0}^{1}\left|x_{1}(t)-x_{2}(t)\right| d t \\
& \leq l_{1}\left\|x_{1}-x_{2}\right\|+M l_{2}\left\|x_{1}-x_{2}\right\| \leq\left(l_{1}+M l_{2}\right)\left\|x_{1}-x_{2}\right\| .
\end{aligned}
$$

Hence

$$
\left[1-\left(l_{1}+M l_{2}\right)\right]\left\|x_{1}-x_{2}\right\| \leq 0,
$$

and then the solution of (7) is unique.

Now, we are in position to state an existence result for the uniqueness of solution for the hybrid implicit functional Equation (1).

Theorem 9. Let the assumptions of Theorems 7 and 8 be satisfied. Then the solution $x \in L_{1}$ of the implicit hybrid delay functional integral Equation (1) is unique.

4.1.1. Continuous Dependence on the Delay Function

Definition 1. The solution $y \in L_{1}$ of the functional integral Equation (6) depends continuously on the function $\varphi$, if $\forall \epsilon>0, \exists \delta>0$, such that

$$
\left|\varphi(t)-\varphi^{*}(t)\right| \leq \delta \Rightarrow\left\|y-y^{*}\right\| \leq \epsilon, t \in I .
$$

Theorem 10. Assume that assumptions of Theorem 7 are verified. Then the solution $y \in L_{1}$ of the Equation (6) depends continuously on $\varphi$.

Proof. Let $y$ be the unique solution of the functional integral Equation (6) and $y^{*}$ is the one of the equation

$$
y^{*}(t)=f_{1}\left(t, y^{*}(t), \int_{0}^{\varphi^{*}(t)} f_{2}\left(t, s, y^{*}(s)\right) d s\right), t \in I .
$$

Then

$$
\begin{aligned}
\left|y(t)-y^{*}(t)\right| & =\mid f_{1}\left(t, y(t), \int_{0}^{\varphi(t)} f_{2}(t, s, y(s)) d s-f_{1}\left(t, y^{*}(t), \int_{0}^{\varphi^{*}(t)} f_{2}\left(t, s, y^{*}(s)\right) d s \mid\right.\right. \\
& \leq b_{1}\left|y(t)-y^{*}(t)\right|+b_{1}\left|\int_{0}^{\varphi(t)} f_{2}(t, s, y(s)) d s-\int_{0}^{\varphi^{*}(t)} f_{2}(t, s, y(s)) d s\right| \\
& +b_{1}\left|\int_{0}^{\varphi^{*}(t)} f_{2}(t, s, y(s)) d s-\int_{0}^{\varphi^{*}(t)} f_{2}\left(t, s, y^{*}(s)\right) d s\right| \\
& \leq b_{1}\left|y(t)-y^{*}(t)\right| \\
& +b_{1} \int_{\varphi^{*}(t)}^{\varphi(t)}\left|f_{2}(t, s, y(s))\right| d s+b_{1} \int_{0}^{\varphi^{*}(t)}\left|f_{2}(t, s, y(s))-f_{2}\left(t, s, y^{*}(s)\right)\right| d s .
\end{aligned}
$$


Now, $\left|\varphi(t)-\varphi^{*}(t)\right| \leq \delta$ and by Lebesgue Theorem [20], we have

$$
\begin{aligned}
\left\|y-y^{*}\right\|_{L_{1}} & \leq b_{1}|| y(t)-y^{*}(t) \|+b_{1} \int_{0}^{1} \int_{\varphi^{*}(t)}^{\varphi(t)}\left|f_{2}(t, s, y(s))\right| d s \\
& +b_{1} b_{2} \int_{0}^{1} \int_{0}^{\varphi^{*}(t)}\left|y(s)-y^{*}(s)\right| d s \\
& \leq b_{1}|| y(t)-y^{*}(t) \|+b_{1} \int_{0}^{1} \epsilon_{1} d s+b_{1} b_{2} \int_{0}^{1} \int_{0}^{1}\left|y(s)-y^{*}(s)\right| d s \\
& \leq \epsilon_{1} b_{1}+\left(b_{1}+b_{1} b_{2}\right)\left\|y-y^{*}\right\|_{L_{1}} .
\end{aligned}
$$

Hence

$$
\left\|y-y^{*}\right\|_{L_{1}} \leq \epsilon_{1} b_{1}\left(1-\left(b_{1}+b_{1} b_{2}\right)\right)^{-1}=\epsilon .
$$

Therefore, $y \in L_{1}$ of the problem (6) depends continuously on $\varphi$. This completes the proof.

4.1.2. Continuous Dependence on the Functions $f_{1}$ and $f_{2}$

Definition 2. (i) The solution $y \in L_{1}$ of the functional integral Equation (6) depends continuously on the function $f_{1}$, if $\forall \epsilon>0, \exists \delta>0$, such that

$$
\begin{gathered}
\left|f_{1}\left(t, y(t), \int_{0}^{\varphi(t)} f_{2}(t, s, y(s)) d s\right)-f_{1}^{*}\left(t, y(t), \int_{0}^{\varphi(t)} f_{2}(t, s, y(s)) d s\right)\right| \leq \delta \\
\Rightarrow\left\|y-y^{*}\right\| \leq \epsilon, t \in I .
\end{gathered}
$$

(ii) The solution $y \in L_{1}$ of Equation (6) depends continuously on the function $f_{2}$, if $\forall \epsilon>0, \exists \delta>0$, such that

$$
\begin{aligned}
\mid f_{1}\left(t, y(t), \int_{0}^{\varphi(t)} f_{2}(t, s, y(s)) d s\right)- & f_{1}\left(t, y(t), \int_{0}^{\varphi(t)} f_{2}^{*}(t, s, y(s)) d s\right) \mid \leq \delta \\
& \Rightarrow\left\|y-y^{*}\right\| \leq \epsilon, t \in I .
\end{aligned}
$$

Theorem 11. Assume that assumptions of Theorem 7 are verified. Then the solution $y \in L_{1}$ of Equation (6) depends continuously on the function $f_{1}$.

Proof. Let $y$ be the unique solution of the functional integral Equation (6) and $y^{*}$ is the solution of the functional integral equation

$$
y^{*}(t)=f_{1}^{*}\left(t, y^{*}(t), \int_{0}^{\varphi(t)} f_{2}\left(t, s, y^{*}(s)\right) d s\right), t \in I .
$$

Then

$$
\begin{aligned}
\left|y(t)-y^{*}(t)\right| & =\left|f_{1}\left(t, y(t), \int_{0}^{\varphi(t)} f_{2}(t, s, y(s)) d s\right)-f_{1}^{*}\left(t, y^{*}(t), \int_{0}^{\varphi(t)} f_{2}\left(t, s, y^{*}(s)\right) d s\right)\right| \\
\leq b_{1}\left|y(t)-y^{*}(t)\right| & +\left|f_{1}\left(t, \int_{0}^{\varphi(t)} f_{2}(t, s, y(s)) d s\right)-f_{1}^{*}\left(t, \int_{0}^{\varphi(t)} f_{2}(t, s, y(s)) d s\right)\right| \\
& +\left|f_{1}^{*}\left(t, \int_{0}^{\varphi(t)} f_{2}(t, s, y(s)) d s\right)-f_{1}^{*}\left(t, \int_{0}^{\varphi(t)} f_{2}\left(t, s, y^{*}(s)\right) d s\right)\right| \\
& \leq b_{1}\left|y(t)-y^{*}(t)\right|+\delta+b_{1} \int_{0}^{\varphi(t)}\left|f_{2}(t, s, y(s))-f_{2}\left(t, s, y^{*}(s)\right)\right| d s \\
& \leq b_{1}\left|y(t)-y^{*}(t)\right|+\delta+b_{1} b_{2} \int_{0}^{\varphi(t)}\left|y(s)-y^{*}(s)\right| d s .
\end{aligned}
$$


Taking supermum for $t \in I$, we have

$$
\begin{aligned}
\left\|y-y^{*}\right\| & \leq b_{1}\left\|y-y^{*}\right\|+\int_{0}^{1} \delta d t+b_{1} b_{2} \int_{0}^{1} \int_{0}^{\varphi(t)}\left|y(s)-y^{*}(s)\right| d s d t \\
& \leq b_{1}\left\|y-y^{*}\right\|+\delta+b_{1} b_{2} \int_{0}^{1} \int_{0}^{1}\left|y(s)-y^{*}(s)\right| d s d t \\
& \leq \delta+\left(b_{1}+b_{1} b_{2}\right)\left\|y-y^{*}\right\| .
\end{aligned}
$$

Now

$$
\left\|y-y^{*}\right\| \leq \frac{\delta}{1-\left(b_{1}+b_{1} b_{2}\right)}=\epsilon .
$$

Hence

$$
|| y-y^{*}|| \leq \epsilon .
$$

Hence, the solution of (6) depends continuously on the function $f_{1}$. This completes the proof.

By the same way we can prove the following theorem.

Theorem 12. Assume that assumptions of Theorem 7 are verified. Then the solution $y \in L_{1}$ of the functional integral Equation (6) depends continuously on the function $f_{2}$.

Definition 3. The solution of functional Equation (7) depends continuously on the function $y$, if $\forall \epsilon>0, \exists \delta>0$, such that

$$
\left\|y-y^{*}\right\| \leq \delta \Rightarrow\left\|x-x^{*}\right\| \leq \epsilon .
$$

Theorem 13. Let the assumptions of Theorem 7 be satisfied. Then the solution of the Equation (7) depends continuously on the function $y$.

Proof. Let $x, x^{*}$ be the solution of the functional Equation (7)

$$
x(t)=h(t, x(t))+y(t) g(t, x(t)), \quad t \in I .
$$

Then

$$
\begin{aligned}
\left|x(t)-x^{*}(t)\right| & =\left|h(t, x(t))+y(t) g(t, x(t))-h\left(t, x^{*}(t)\right)-y^{*}(t) g\left(t, x^{*}(t)\right)\right| \\
& \leq\left|h(t, x(t))-h\left(t, x^{*}(t)\right)\right|+|g(t, x(t))|\left|y(t)-y^{*}(t)\right| \\
& +\left|y^{*}(t)\right|\left|g(t, x(t))-g\left(t, x^{*}(t)\right)\right| \\
& \leq l_{1}\left|x(t)-x^{*}(t)\right|+\left[l_{2}|x(t)|+G\right]\left|y(t)-y^{*}(t)\right|+l_{2}\left|y^{*}(t)\right|\left|x(t)-x^{*}(t)\right| .
\end{aligned}
$$

Then for $t \in I,|x(t)|<N$ and $\left|y^{*}(t)\right|<M$, we have

$$
\begin{aligned}
& \left\|x_{1}-x_{2}\right\| \\
& \leq l_{1} \int_{0}^{1}\left|x(t)-x^{*}(t)\right| d t+\left[l_{2} N+G\right] \int_{0}^{1}\left|y(t)-y^{*}(t)\right| d t+l_{2} M \int_{0}^{1}\left|x(t)-x^{*}(t)\right| d t \\
& \leq l_{1}\left\|x-x^{*}\right\|_{L_{1}}+\left[\mathrm{t}_{2} N+G\right]\left\|y-y^{*}\right\|_{L_{1}}+l_{2} M\left\|x-x^{*}\right\|_{L_{1}} \\
& \leq l_{1}\left\|x-x^{*}\right\|_{L_{1}}+\left[\mathrm{t}_{2} N+G\right] \delta+l_{2} M\left\|x-x^{*}\right\|_{L_{1}}
\end{aligned}
$$

Hence

$$
\left\|x-x^{*}\right\|_{L_{1}} \leq \delta\left(\mathrm{t}_{2} N+G\right)\left[1-\left(l_{1}+l_{2} M\right)\right]^{-1}=\epsilon .
$$

Hence the solution of problem (7) depends continuously on the function $y$. 
Now, from Theorems 10-13, we have the following corollaries

Corollary 1. The solution of the hybrid implicit integral Equation (1) depends continuously on the delay function $\varphi$.

Corollary 2. The solution of the the hybrid implicit integral Equation (1) depends continuously on a function $f_{1}$.

Corollary 3. The solution of the the hybrid implicit integral Equation (1) depends continuously on a function $f_{2}$.

\section{Special Cases and Examples}

We can deduce the following particular cases.

- $\quad$ Taking $\varphi(t)=t$, then we have

$$
\frac{x(t)-h(t, x(t))}{g(t, x(t))}=f_{1}\left(t, \frac{x(t)-h(t, x(t))}{g(t, x(t))}, \int_{0}^{t} f_{2}\left(t, s, \frac{x(s)-h(s, x(s))}{g(s, x(s))}\right) d s\right), t \in I .
$$

- $\quad$ For $g(t, x)=1$, the following equation is obtained

$$
\left(x(t)-h(t, x(t))=f_{1}\left(t,\left(x(t)-h(t, x(t)), \int_{0}^{t} f_{2}(t, s,(x(s)-h(s, x(s))) d s), t \in I .\right.\right.\right.
$$

- For $h(t, x)=0$, the following equation is obtained

$$
\frac{x(t)}{g(t, x(t))}=f_{1}\left(t, \frac{x(t)}{g(t, x(t))}, \int_{0}^{\varphi(t)} f_{2}\left(t, s, \frac{x(s)}{g(s, x(s))}\right) d s\right), t \in I .
$$

Furthermore, taking $f_{1}(t, x, y)=f_{1}(t, x)$ and $g(t, x)=1$, in Equation (14), then we have the functional equation, which is studied in [6].

- Putting $f_{1}(t, x, y)=a(t)+x(t) \cdot y(t)$ and $g(t, x)=1$, in Equation (14), then we have the quadratic Urysohn integral equation

$$
x(t)=a(t)+x(t) \int_{0}^{\varphi(t)} f_{2}(t, s, x(s)) d s, \quad t \in I,
$$

where $a \in L_{1}(I)$.

- Putting $f_{1}(t, x, y)=a(t)+y(t)$ and $g(t, x)=1$, in Equation (14), then we have the Urysohn integral equation

$$
x(t)=a(t)+\int_{0}^{\varphi(t)} f_{2}(t, s, x(s)) d s, t \in I,
$$

where $a \in L_{1}(I)$ and taking $\varphi(t)=t$, which is the same result obtained in [7]

Example 1. Consider a nonlinear implicit hybrid functional integral equation

$$
\begin{aligned}
& \frac{x(t)-\left[\frac{e^{-t}}{20+t^{2}}+\frac{1}{2} \sqrt{x(t)}\right]}{\frac{1}{\sqrt{16+t}}+\frac{e^{-t}}{200} x(t)} \\
= & \frac{1}{2}\left(\int_{0}^{t}\left[e^{-(t+s)} \sqrt{\left(\frac{x(s)-\left[\frac{e^{-s}}{20+s^{2}}+\frac{1}{2} \sqrt{|x(s)|}\right]}{\frac{1}{\sqrt{16+s}}+\frac{e^{-s}}{200} x(s)}\right)}+\frac{s}{2(s+1)}\right] d s\right) \\
& +\frac{x(t)-\left[\frac{e^{-t}}{20+t^{2}}+\frac{1}{2} \sqrt{x(t)}\right]}{2\left(\frac{1}{\sqrt{16+t}}+\frac{e^{-t}}{200}|x(t)|\right)}+\frac{1}{2 e^{t}} .
\end{aligned}
$$


Set

$$
\begin{aligned}
h(t, x(t)) & =\frac{e^{-t}}{20+t^{2}}+\frac{1}{2} \sqrt{x(t)}, \\
g(t, x(t)) & =\frac{1}{\sqrt{16+t}}+\frac{e^{-t}}{200} x(t), \\
f_{1}(t, y(t), z(t)) & =\frac{1}{2}\left(\frac{1}{e^{t}}+y(t)+z(t)\right)
\end{aligned}
$$

where

$$
\begin{aligned}
& y(t)=\frac{x(t)-\left[\frac{e^{-t}}{20+t^{2}}+\frac{1}{2} \sqrt{x(t)}\right]}{\frac{1}{\sqrt{16+t}}+\frac{e^{-t}}{200} x(t)}, \\
& z(t)=\int_{0}^{t} f_{2}(t, s, y(s)) d s=\int_{0}^{t}\left[\frac{1}{e^{t+s}} \sqrt{y(s)}+\frac{s}{2(s+1)}\right] d s .
\end{aligned}
$$

It is easy to obtain that

$$
\begin{gathered}
\left|f_{1}(t, y(t), z(t))\right| \leq \frac{1}{2}\left(\frac{1}{e}+|y(t)|+|z(t)|\right), \\
\left|f_{2}(t, s, x(s))\right| \leq e^{-t} \sqrt{x(s)}+\frac{s}{2(s+t)}, \\
|h(t, x(t))| \leq \frac{1}{20}+\frac{x(t)}{2}, \\
|g(t, x(t))| \leq \frac{1}{4}+\frac{x(t)}{200} .
\end{gathered}
$$

By setting

$$
\begin{gathered}
m_{1}(t)=\frac{1}{2} x(t), \quad m_{2}(t)=\frac{1}{e^{t}} \sqrt{x(t)}, a_{1}(t)=\frac{e^{-t}}{20+t^{2}}, \\
a_{2}(t)=\frac{1}{\sqrt{16+t}} \in L_{1}[0,1], \quad b_{1}=\frac{1}{2 e}, b_{2}=\frac{1}{2}, \quad l_{1}=\frac{1}{2}, \quad l_{2}=\frac{1}{200}, \quad M=\frac{1}{e}
\end{gathered}
$$

we can find that

$$
l_{1}+M l_{2}=0.5018393972<1 \text { and } b_{1} b_{2}=\frac{1}{4 e}<1 .
$$

The conclusion of Theorem 6 can be implied that the implicit hybrid functional integral Equation (15) has at least one solution.

Example 2. Consider a nonlinear implicit hybrid functional integral equation

$$
\frac{x(t)-\frac{5 e^{t}}{7} \frac{x(t)}{1+|x(t)|}}{e^{-\pi t}+\frac{e^{-\ln (t+1) x(t)}}{1+|x(t)|}}=\frac{1}{60} e^{t}\left(\frac{x(t)-\frac{5 e^{t}}{7} \frac{x(t)}{1+|x(t)|}}{e^{-\pi t}+\frac{e^{-\ln (t+1) x(t)}}{1+|x(t)|}}+\int_{0}^{t} \frac{1}{e^{t+s}}\left(\frac{x(t)-\frac{5 e^{t}}{7} \frac{x(t)}{1+|x(t)|}}{e^{-\pi t}+\frac{e^{-\ln (t+1) x(t)}}{1+|x(t)|}}\right) d s\right) .
$$

Set

$$
\begin{aligned}
h(t, x) & =\frac{5 e^{t}}{7} \frac{x(t)}{1+|x(t)|^{\prime}} \\
g(t, x) & =e^{-\pi t}+\frac{e^{-\ln (t+1)} x(t)}{1+|x(t)|}, \\
f_{1}(t, y(t), z(t)) & =\frac{1}{60} e^{t}(y(t)+z(t)),
\end{aligned}
$$


where

$$
\begin{aligned}
& y(t)=\frac{x(t)-\frac{5 e^{t}}{7} \frac{x(t)}{1+|x(t)|}}{e^{-\pi t}+\frac{e^{-\ln (t+1) x(t)}}{1+|x(t)|}} \\
& z(t)=\int_{0}^{t} f_{2}(t, s, y(s)) d s=\int_{0}^{t} e^{-(t+s)} y(s) d s .
\end{aligned}
$$

It is easy to obtain that

$$
\begin{gathered}
\left|f_{1}\left(t, y_{1}, z_{1}\right)-f_{1}\left(t, y_{2}, z_{1}\right)\right| \leq \frac{1}{60} e^{t}\left(\left|y_{1}-y_{2}\right|+\left|z_{1}-z_{2}\right|\right), \\
\left|f_{2}\left(t, s, y_{1}\right)-f_{2}\left(t, s, y_{2}\right)\right| \leq \frac{1}{e^{2}}\left|y_{1}-y_{2}\right| \\
\left|h\left(t, x_{1}\right)-h\left(t, x_{2}\right)\right| \leq \frac{5 e^{t}}{7}\left|x_{1}-x_{2}\right|
\end{gathered}
$$

and

$$
\left|g\left(t, x_{1}\right)-g\left(t, x_{2}\right)\right| \leq e^{-\ln (t+1)}\left|x_{1}-x_{2}\right|,
$$

for all $x_{1}, x_{2} \in \mathbb{R}$ and $t \in[0,1]$.

If

$$
l_{1}(t)=e^{-\ln (t+1)}, l_{2}(t)=\frac{5 e^{t}}{7}, \quad b_{1}(t)=\frac{1}{60} e^{t}, \quad b_{2}(t)=e^{-(t+s)},
$$

then

$$
l_{1}=\frac{1}{2}, l_{2}=\frac{5 e}{7} \text { and } b_{1}=\frac{e}{60}, b_{2}=\frac{1}{e^{2}} .
$$

Hence, conditions $b_{1} b_{2}=\frac{1}{60 e} \approx 0.00613<1$ and $l_{1}+M l_{2} \approx 0.8151174656<1$, holds.

Now by using Theorem 9, the implicit hybrid integral Equation (16) has unique solution.

\section{Conclusions}

The solvability of functional equations have gained much attention. Many researchers have obtained existence results in different classes. In this work, we have proved the existence of nondecreasing a.e. integrable solutions of an implicit hybrid delay nonlinear functional equation. The technique of measures of noncompactness is utilized to obtain our main result. A sufficient condition is presented for the uniqueness of the solution. The continues dependence of solution on the delay function $\varphi$ and on the functions $f_{1}$ and $f_{2}$ have been obtained. Additionally, some particular cases and examples are given to support the main results.

Author Contributions: Conceptualization, A.M.A.E.-S., H.H.G.H. and S.M.A.-I. All authors have read and agreed to the published version of the manuscript.

Funding: This research received no external funding.

Institutional Review Board Statement: Not applicable.

Informed Consent Statement: Not applicable.

Acknowledgments: Authors are grateful to referees for their useful comments and remarks that helped to improve this work.

Conflicts of Interest: The authors declare no conflict of interest.

\section{References}

1. Nieto, J.J.; Ouahab, A.; Venktesh, V. Implicit fractional differential equations via the Liouville Caputo derivative. Mathematics 2015, 3, 398-411. [CrossRef]

2. Benchohra, M.; Souid, M. Integrable solutions for implicit fractional order differential equations. TJMM 2014, 6, 101-107. 
3. Dhage, B.C.; Lakshmikantham, V. Basic results on hybrid differential equation. Nonlinear Anal. Hybrid Syst. $2010,4,414-424$. [CrossRef]

4. Zhao, Y.; Sun, S.; Han, Z.; Li, Q. Theory of fractional hybrid differential equations. Comput. Math. Appl. 2011, 62, 1312-1324. [CrossRef]

5. Sun, S.; Zhao, Y.; Han, Z.; Li, Y. The existence of solutions for boundary value problem of fractional hybrid differential equations Commun. Nonlinear Sci. Numer. Simul. 2012, 17, 4961-4967. [CrossRef]

6. Banaś, J. On the superposition operator and integrable solutions of some functional equations. Nonlinear Anal. 1988, 12, 777-784. [CrossRef]

7. Banaś, J. Integrable solutions of Hammerstein and Urysohn integral equations. J. Austral. Math. Soc. (Ser. A) 1989, 46, 61-68. [CrossRef]

8. El-Sayed, A.M.A.; Al-Issa, S.M. Monotonic continuous solution for a mixed type integral inclusion of fractional order. J. Math. Appl. 2010, 33, 27-34.

9. El-Sayed, A.M.A.; Al-Issa, S.M. Monotonic integrable solution for a mixed type integral and differential inclusion of fractional orders. Int. J. Differ. Equ. Appl. 2019, 18,1-9.

10. El-Sayed, A.M.A.; Hashem, H.H.G. Integrable and continuous solutions of nonlinear quadratic integral equation. Electron. J. Qual. Theory Differ. Equ. 2008, 25, 1-10. [CrossRef]

11. El-Sayed, A.M.A.; Hashem, H.H.G. Monotonic solutions of functional integral and differential equations of fractional order. Electron. J. Qual. Theory Differ. Equ. 2009, 2009, 1-8. [CrossRef]

12. El-Sayed, A.M.A.; Hashem, H.H.G.; Al-Issa, S.M. Characteristics of solutions of fractional hybrid integro-differential equations in Banach Algebra. Sahand Commun. Math. Anal. 2021, 18, 109-131.

13. Salem, A.; Alshehri, H.M.; Almaghamsi, L. Measure of noncompactness for an infinite system of fractional Langevin equation in a sequence space. Adv. Differ. Equ. 2021, 2021, 132. [CrossRef]

14. Salem, A.; Al-Dosari, A. Positive Solvability for Conjugate Fractional Differential Inclusion of $(k, n-k)$ Type without Continuity and Compactness. Axioms 2021, 10, 170. [CrossRef]

15. Srivastava1, H.M.; El-Sayed, A.M.A.; Hashem, H.H.G.; Al-Issa, S.M. Analytical investigation of nonlinear hybrid implicit functional differential inclusions of arbitrary fractional orders. Racsam 2022, 116, 26. [CrossRef]

16. Blasi, F.S.D. On a property of the unit sphere in a Banachspace. Bull. Math. Soc. Sci. 1977, 21, 259-262.

17. Appell, J.; Pascale, E.D. Su alcuni parametri connessi con la misura di non compattezza di Hausdorff in spazi di funzioni misurabili. Boll. Un. Mat. Ital. 1984, 6, 497-515.

18. Banaś, J.; Goebel, K. Measures of Noncompactness in Banach Spaces; Lecture Notes in Pure and Applied Mathematics; Marcel Dekker: New York, NY, USA, 1980; Volume 60.

19. Natanson, I.P. Theory of Functions of a Real Variable; Ungar: New York, NY, USA, 1960.

20. Kolmogorov, A.N.; Fomin, S.V. Introductory Real Analysis; Courier Corporation: North Chelmsford, MT, USA, 1975. 